\title{
Optimality conditions for set-valued optimization problems via scalarization function
}

\author{
Ha Manh Linh ${ }^{1,}{ }^{*}$, Nguyen Dinh Huy ${ }^{2}$, Nguyen Thi Thanh Truc ${ }^{1}$
}

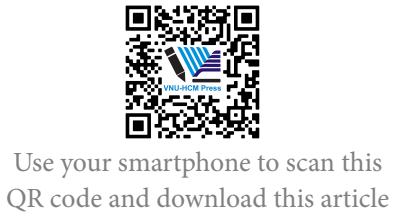

${ }^{1}$ University of Information

Technology, VNU-HCM

${ }^{2}$ University of Technology, VNU-HCM

Correspondence

Ha Manh Linh, University of Information Technology, VNU-HCM

Email: hamanhlinh2002@gmail.com

History

- Received: 09-12-2019

- Accepted: 17-12-2020

- Published: 31-12-2020

DOI : 10.32508/stdjet.v3iSI3.642

\section{Check for updates}

Copyright

(c) VNU-HCM Press. This is an openaccess article distributed under the terms of the Creative Commons Attribution 4.0 International license.

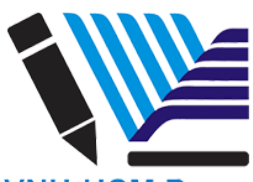

VNU-HCM Press

\begin{abstract}
One of the most important and popular topics in optimization problems is to find its optimal solutions, especially Pareto optimal points, a well-known solution introduced in multi-objective optimization. This topic is one of the oldest challenges in many issues related to science, engineering and other fields. Many important practical-problems in science and engineering can be expressed in terms of multi-objective/ set-valued optimization problems in order to achieve the proper results/ properties. To find the Pareto solutions, a corresponding scalarization problem has been established and studied. The relationships between the primal problem and its scalarization one should be investigated for finding optimal solutions. It can be shown that, under some suitable conditions, the solutions of the corresponding scalarization problem have uniform spread and have a close relationship to Pareto optimal solutions for the primal one. Scalarization has played an essential role in studying not only numerical methods but also duality theory. It can be usefully applied to get relationships/ important results between other fields, for example optimization, convex analysis and functional analysis. In scalarization, we ussually use a kind of scalarized-functions. One of the first and the most popular scalarized-functions used in scalarization method is the Gerstewitz function. In the paper, we mention some problems in set-valued optimization. Then, we propose an application of the Gerstewitz function to these problems. In detail, we establish some optimality conditions for Pareto/ weak solutions of unconstrained/ constrained set-valued optimization problems by using the Gerstewitz function. The study includes the consideration of problems in theoretical approach. Some examples are given to illustrate the obtained results.
\end{abstract}

Key words: Pareto efficient solution, weak efficient solution, set-valued optimization, Gerstewitz function, optimality condition

\section{INTRODUCTION}

Scalarization has an essential role in studying numerical methods and duality theory ${ }^{1-4}$. It can be applied to get relationships between other fields, such as: optimization, convex analysis and functional analysis. Solutions of vector optimization problems can be characterized by those of corresponding scalarized optimization problems.

In scalarization, the Gerstewitz function plays an important role. Its main properties were studied in some papers $^{5-7}$.

In the paper, we have proposed optimality conditions for set-valued optimization problems using the Geraterwitz function. These results have contributed to applications of the Gerstewitz function in optimization.

\section{RELIMINARIES}

In the paper, Let $X$ and $Y$ be normed spaces, $K$ be a pointed, closed, convex cone witn nonempty interior in $Y$. For $A$ be a nonempty subset in $Y$, we denote
$\operatorname{int}(A), \operatorname{cl}(A)$ and $\operatorname{cone}(A)$ for the interior, the closure of $A$ and the cone generalized by $A$, respectively.

Let $F:=X \rightarrow 2^{Y}$ be a set-valued map from $X$ to $Y$, the domain, the image and the graph of $F$ are defined by $\operatorname{dom}(F) \quad:=\{x \in X \mid F(x) \neq \varnothing\} ; \quad \operatorname{im}(F) \quad:=$ $\{y \in Y \mid y \in F(\operatorname{dom}(F))\}$

$\operatorname{gr}(F):=\{(x, y) \in X \times Y \mid y \in F(x)\}$.

Definition 2.1. Let $F:=X \rightarrow 2^{Y}$ and $\left(x_{0}, y_{0}\right) \in$ $\operatorname{gr}(F)$.

(i) A point $\left(x_{0}, y_{0}\right)$ is called a Pareto efficient solution of $F$ on $X$ if $\left(F(X)-y_{0}\right) \cap(-K \backslash\{0\})=\varnothing$. The set of Pareto efficient solutions of $F$ is denoted by $\operatorname{Min}_{K}$ $F(X)$.

(ii) A point $\left(x_{0}, y_{0}\right)$ is called a weak efficient solution of $F$ on $X$ if $\left(F(X)-y_{0}\right) \cap(-\operatorname{int} K)=\varnothing$. The set of weak efficient solutions of $F$ is denoted by $\operatorname{WMin}_{K}$ $F(X)$.

Note that $\operatorname{Min}_{K} F(X)$ is a subset of $\operatorname{WMin}_{K} F(X)$. In general, the inverse conclusion is not true by the following example.

Example 2.2. Let $X=\mathbb{R}, Y=\mathbb{R}^{2}, k=\mathbb{R}_{+}^{2}, F_{1}, F_{2}$ : $X \rightarrow 2^{Y}$ be defined by 
$F_{1}(x):=\left\{\left(y_{1}, y_{2} \in Y \mid y_{2} \geq y_{1}^{2}\right)\right\} ;$

$F_{2}(x):=\left\{\left(y_{1}, y_{2} \in Y \mid y_{2} \geq y_{1}^{2}, y_{1} \leq 0\right)\right\} \cup$

$\left\{\left(y_{1}, y_{2}\right) \in Y \mid y_{2} \geq 0, y_{1} \geq 0\right\}$.

With $F_{1}$, one has $\operatorname{Min}_{K} F_{1}(X) \quad \operatorname{Wmin}_{K} F_{1}(X)$ $=\left\{\left(x\left(y_{1}, y_{2}\right)\right) \in X \times Y \mid y_{2}=y_{1}^{2}, y_{1} \leq 0\right\}$.

With $F_{2}$, we get

$\operatorname{Min}_{K} F_{2}(X)=\left\{\left(x\left(y_{1}, y_{2}\right)\right) \in X \times Y \mid y_{2}=y_{1}^{2}, y_{1} \leq 0\right\}$, $\operatorname{Wmin}_{K} F_{2}(X)=\left\{\left(x\left(y_{1}, y_{2}\right)\right) \in X \times Y \mid y_{2}=y_{1}^{2}, y_{1} \leq 0\right\} \cup$ $\left\{\left(x\left(y_{1}, y_{2}\right)\right) \in X \times Y \mid y_{2}=0, y_{1} \geq 0\right\}$. Therefore, $\operatorname{Min}_{K} F_{2}(X)$ is a subset of $\operatorname{Wmin}_{K} F_{2}(X)$.

Definition 2.3. ${ }^{6}$ With $e \in \operatorname{int}(K)$, the Gerstewitz function $h_{K}^{e}(y): Y \rightarrow \mathbb{R}$ is defined by $h_{K}^{e}(y):=\inf \{t \in R \mid y \in t e-K\}$.

If $K$ is a pointed, closed, convex cone with nonempty interior then $h_{K}^{e}$ is finite and we get $h_{K}^{e}(y)=\sup \{h(y) \mid h \in K *, h(e)=1\}, \forall y \in Y$, where $K *:=\{k * \in Y * \mid\langle k *, k\rangle \geq 0, \forall k \in K\}$. It is a convex and continuous function on $Y$. We recall some properties of the Gerstewitz function as follows.

Proposition 2.4. ${ }^{6,7}$ With $e \in \operatorname{int}(K)$, we have

(i) $(-\infty, 0] e-K=\left\{y \in Y \mid h_{K}^{e}(y) \leq 0\right\}$.

(ii) $(-\infty, 0) e-K=\left\{y \in Y \mid h_{K}^{e}(y)<0\right\}$.

(iii) $-K \backslash((-\infty, 0) e-K)=\left\{y \in Y \mid h_{K}^{e}(y)=0\right\}$.

(iv) $Y \backslash((-\infty, 0) e-K)=\left\{y \in Y \mid h_{K}^{e}(y) \geq 0\right\}$.

(v) $Y \backslash((-\infty, 0] e-K)=\left\{y \in Y \mid h_{K}^{e}(y)>0\right\}$.

(vi) $\forall \alpha>0, h_{K}^{e}(\alpha y)=\alpha h_{K}^{e}(y)$.

(vii) $h_{K}^{e}\left(y_{1}+y_{2}\right) \leq h_{K}^{e}\left(y_{1}\right)+h_{K}^{e}\left(y_{2}\right)$.

For applications of the Garsterwitz function, the readers are reffered to the references ${ }^{6-9}$.

\section{OPTIMALITY CONDITIONS}

Let $X, Y, Z$ be normed spaces, $K$ and $D$ be pointed, closed, convex cones with nonempty interior in $Y$ và $Z$, respectively, $F: X \rightarrow 2^{Y}, G: X \rightarrow 2^{Z}$. We consider two optimization problems as follows

$$
\begin{gathered}
\left(P_{1}\right)=\left\{\begin{array}{l}
\min _{K} F(x), \\
\text { s.t. } x \in X ;
\end{array}\right. \\
\left(P_{2}\right)=\left\{\begin{array}{l}
\min _{K} F(x), \\
\text { s.t. }, x \in X, G(x) \cap(-D) \neq \varnothing .
\end{array}\right.
\end{gathered}
$$

Let $S_{i}$ be feasible sets of $\left(P_{i}\right), i=1,2$, then

$S_{1}=X$ and $S_{2}=\{x \in X \mid G(x) \cap(-D) \neq \varnothing\}$

A point $\left(x_{0}, y_{0}\right) \in \operatorname{gr}(F)$ is called a Pareto efficient solution (a weak efficient solution) of $\left(P_{i}\right)(i=1,2)$ if $x_{0} \in S_{i}$ and

$\left(F\left(S_{i}\right)-y_{0}\right) \cap(-K \backslash\{0\})=\varnothing$.

$\left(F\left(S_{i}\right)-y_{0}\right) \cap(-\operatorname{int} K)=\varnothing$.

Theorem 3.1. Let $\left(x_{0}, y_{0}\right) \in \operatorname{gr}(F)$ with $x_{0} \in S_{1}$. Then, $\left(x_{0}, y_{0}\right)$ is a weak efficient solution of $\left(P_{1}\right)$ if and only if there exists int $(K)$ such that $\forall y \in F\left(S_{1}\right)$,

$$
h_{K}^{e}\left(y-y_{0}\right) \geq 0 .
$$

Proof. "If:" Suppose that there exists $e \in \operatorname{int}(K)$ such that $h_{K}^{e}\left(y-y_{0}\right) \geq 0, \forall y \in F\left(S_{1}\right)$. Let $v \in F\left(S_{1}\right)-y_{0}$, then $y \in F\left(S_{1}\right)$ there is with $v=y-y_{0}$. Because (1) is fulfilled, i.e., $h_{K}^{e}(v) \geq 0$, by Proposition 2.4(iv) we obtain $v \in Y \backslash((-\infty, 0) e-K)$, thus $v \in Y \backslash(-$ int $K)$. Consequently, one gets

$\left(F\left(S_{1}\right)-y_{0}\right) \cap(-\operatorname{int} K)=\varnothing$.

Therefore, $\left(x_{0}, y_{0}\right)$ a weak efficient solution of $\left(P_{1}\right)$.

"Only if:" Let $\left(x_{0}, y_{0}\right)$ be a weak efficient solution of $\left(P_{1}\right)$, suppose that $(1)$ does not hold, i.e., there exist $x \in S_{1}, y \in F\left(S_{1}\right)$ with $h_{K}^{e}\left(y-y_{0}\right)<0$ By Proposition 2.4(ii), we get $y-y_{0} \in(-\infty, 0) e-K=-\operatorname{int} K$, which contradicts to the weak efficiency of $\left(x_{0}, y_{0}\right)$.

Theorem 3.2. Let $\left(x_{0}, y_{0}\right) \in \operatorname{gr}(F)$ with $x_{0} \in S_{1}$. (i) If $\left(x_{0}, y_{0}\right)$ is a Pareto efficient soluton of (P1) then there exists $e \in \operatorname{int}(K)$ such that (1) holds $\forall y \in F\left(S_{1}\right)$. (ii) If there exists $e \in \operatorname{int}(K)$ such that (1) holds with strict inequality $\forall y \in F\left(S_{1}\right) \backslash\left\{y_{0}\right\}$ then $\left(x_{0}, y_{0}\right)$ is a Pareto efficient solution of (P1).

Proof. (i) Since $\left(x_{0}, y_{0}\right)$ is a Pareto efficient solution of $\left(P_{1}\right),\left(x_{0}, y_{0}\right)$ is a weak efficient solution of $\left(P_{1}\right)$. It follows from Theorem 3.1 that we are done.

(ii) Suppose that there is $e \in \operatorname{int}(K)$ with $h_{K}^{e}\left(y-y_{0}\right)>$ $0, \forall y \in F\left(S_{1}\right) \backslash\left\{y_{0}\right\}$. Let $v \in F\left(S_{1}\right)-y_{0}$, then there exists $y \in F\left(S_{1}\right)$ such that $v=y-y_{0}$. Since (1) holds with strict inequality, i.e., $h_{K}^{e}(v)>0$ by Proposition 2.4(v), we get $v \in Y \backslash((-\infty, 0) e-K)$, so $v \in Y \backslash(-K)$. Therefore, we get

$\left(F\left(S_{1}\right)-y_{0}\right) \cap(-K \backslash\{0\})=\varnothing$.

Hence, $\left(x_{0}, y_{0}\right)$ is a Pareto efficient solution of $\left(P_{1}\right)$.

With the problem $\left(P_{2}\right)$, we can obtain optimality conditions simiar to those in Theorem 3.1 and 3.2 replacing $S_{1}$ by $S_{2}$. However, since $\left(P_{2}\right)$ is a constrained optimization problem, we propose other results for this problem.

Let $O(Z, Y)$ be a set of continuous maps from $Z$ to $Y$, and

$\Pi:=\{T \in O(Z, Y) \mid T(D) \subseteq K\}$

We define the map $L: X \times \Pi \rightarrow 2^{Y}$ by $L(x, T):=$ $F(x)+T(G(x))$.

Theorem 3.3. Let $\left(x_{0}, y_{0}\right) \in \operatorname{gr}(F)$ with $x_{0} \in S_{2}$ and $z_{0} \in G(x) \cap(-D)$. Then, $\left(x_{0}, y_{0}\right)$ is a weak efficient solution of (P2) if and only if there exist $e \in \operatorname{int}(K), T \in \Pi$ such that $\forall x \in X, y \in L(x, T)$,

$$
h_{K}^{e}\left(y-y_{0}\right) \geq 0 \text {. }
$$

Proof. "If:" Since (2) holds, by Proposition 2.4(iv), one gets $L(x, T)-y_{0} \subseteq Y \backslash((-\infty, 0) e-K)=Y \backslash(-\operatorname{int} K)$, or

$$
F(x)+T(G(x))-y_{0} \subseteq Y \backslash(-\operatorname{int} K)
$$


Suppose that $\left(x_{0}, y_{0}\right)$ is not a weak efficient solution of $\left(P_{2}\right)$, there exist $x \in \widetilde{X}, z \in \widetilde{G}(x) \cap(-\widetilde{D}), y \in \widetilde{F}(x)$ such that $y-y_{0} \in-\operatorname{int} K$. Therefore,

$y+\widetilde{T}(z)-y_{0} \in-\operatorname{int} K-K=-\operatorname{int} K$,

which contradicts to (3). Hence, $\left(x_{0}, y_{0}\right)$ is a weak efficient solution of $\left(P_{2}\right)$.

"Only if:" Suppose that (2) does not hold, i.e., for all $e \in \operatorname{int}(K), T \in \Pi$ one has $x \in X$ such that $h_{K}^{e}\left(L(x, T)-y_{0}\right)<0$. By Proposition 2.4(ii), we obtain

$L(x, T)-y_{0} \subseteq(-\infty, 0) e-K \subseteq-\operatorname{int} K$

It means that $F(x)+T(G(x))-y_{0} \subseteq-\operatorname{int} K$. For $\mathrm{x}$ with $\widetilde{G}(x) \cap(-D) \neq \varnothing$, there is $z \in \widetilde{G}(x) \cap(-\widetilde{D}), y \in$ $\widetilde{F}(x)$ such that $y-y_{0} \in-\operatorname{int} K$, which is a contradiction. Hence, (2) is fulfilled.

Theorem 3.4. Let $\left(x_{0}, y_{0}\right) \in \operatorname{gr}(F)$ with $x_{0} \in S_{2}$ and $z_{0} \in G\left(x_{0}\right) \cap(-D)$.

(i) If $\left(x_{0}, y_{0}\right)$ is a Pareto efficient solution of (P2) then there exist $e \in \operatorname{int}(K)$, such that (2) holds $\forall x \in X, y \in$ $L(x, T)$.

(ii) If there exist $e \in \operatorname{int}(K), T \in \Pi$ such that (2) holds with strict inequality $\forall x \in X, y \in L(x, T) \backslash\left\{y_{0}\right\}$ then $\left(x_{0}, y_{0}\right)$ is a Pareto efficient solution of $(P 2)$.

Proof. (i) Since $\left(x_{0}, y_{0}\right)$ is a Pareto efficient solution of $\left(P_{2}\right),\left(x_{0}, y_{0}\right)$ is a weak efficient solution of $\left(P_{2}\right)$. By Theorem 3.3, we are done.

(ii) Since (2) holds with strict inequality, by Proposition $2.4(\mathrm{v})$, one gets $L(x, T)-y_{0} \subseteq((-\infty, 0] e-K)=$ $Y \backslash(-K)$, or (4).

$$
F(x)+T(G(x))-y_{0} \subseteq Y \backslash(-K) .
$$

Suppose that $\left(x_{0}, y_{0}\right)$ is not a Pareto efficient solution of $\left(P_{2}\right)$, there exist $x \in \widetilde{X}, z \in \widetilde{G}(x) \cap(-\widetilde{D}), y \in \widetilde{F}(x)$ such that $y-y_{0} \in-K \backslash\{0\}$. Therefore, $y+\widetilde{T}(z)-y_{0} \in-K \backslash\{0\}-K \subseteq-K$,

which contradicts to $(4)$. Hence, $\left(x_{0}, y_{0}\right)$ is a Pareto efficient solution of $\left(P_{2}\right)$.

To illustrate Theorem 3.3, we consider the following example.

Example 3.5. Let $X=Y=Z=\mathbb{R}, K=D=\mathbb{R}_{+}, F$ : $X \rightarrow 2^{Y}, G: X \rightarrow 2^{Z}$ be defined by

$F(x) \quad:=\quad\left\{y \in Y \mid 0 \leq y \leq x^{2}\right\} ; \quad G(x) \quad:=$ $\{z \in Z|0 \leq z \leq| x \mid\}$.

With $\left(P_{2}\right)$, one has $\{x \in X \mid G(x) \cap(-D) \neq \varnothing\}=\mathbb{R}$. It is easy to see that $\left(x_{0}, y_{0}\right)=(0,0)$ is a weak efficient solution of $\left(P_{2}\right)$. We now check that condition (2) holds. In fact, with $e=1, T(x)=x$ is a linear operator satisfying $T(D) K$, then

$L(x, T):=F(x)+T(G(x))=\left[0, x^{2}\right]+[0,|x|]=$ $\left[0, x^{2}+|x|\right]$.

For all $y \in L(x, T), x \in X$, one gets

$h_{K}^{e}\left(y-y_{0}\right):=\inf \{t \in \mathbb{R} \mid y \in t e-K\}$

$=\inf \left\{t \in \mathbb{R} \mid\left[0, x^{2}+|x|\right] \in t-K\right\}$

$=x^{2}+|x| \geq 0$.

\section{CONCLUSIONS}

In the paper, we first recall the Gerstewitz scalar function and its basic properties. Via this function, optimality conditions for some kinds of optimization problems are established concerning Pareto efficient/ weak efficient solutions.

In set-valued optimization, we have several kinds of solutions, such as: Geoffrion efficient solution ${ }^{10}$, Borwein efficent solution ${ }^{11}$, Benson efficient solution $^{12}$... They have been studied in some recent results $^{13,14}$. Thus, for the possible development, we think that giving optimality conditions for the abovementioned solutions may be a promising approach.

\section{AUTHOR S' CONTRIBUTION}

All authors contributed equally to this work. All authors have read and agreed to the published version of the manuscript.

\section{CONFLICT OF INTEREST}

We declare that there is no conflict of whatsoever involved in publishing this research.

\section{ACKNOWLEDGMENT}

This rearch is funded by Vietnam National University of Hochiminh City (VNU HCMC) under grant number C2019-26-03.

\section{REFERENCES}

1. Adan M, Novo V. Proper efficiency in vector optimization on real linear spaces, J. Optim. Theory Appl. 2004;121:515-540. Available from: https://doi.org/10.1023/B:JOTA.0000037602. 13941.ed.

2. Jahn J. Vector optimization. Theory, Applications, and Extensions. Berlin: Springer. 2011;

3. Zhou ZA, Peng JW. Scalarization of set-valued optimization problems with generalized cone subconvexlikeness in real ordered linear spaces. J. Optim. Theory Appl. 2012;154:830-841. Available from: https://doi.org/10.1007/s10957-012-0045-2.

4. Zhou ZA, Yang XM, Peng JW. Scalarization of -super efficient solutions of set-valued optimization problems in real ordered linear spaces. J. Optim. Theory Appl. 2014;162:680-693. Available from: https://doi.org/10.1007/s10957-014-0565-z.

5. Chen GY, Huang XX, Yang XG. Vector optimization: Set-valued and variational analysis. Berlin: Springer-Verlag. 2005;

6. Gutirréez C, Novo V, Rodenas-Pedregosa JL, Tanaka T. Nonconvex separation functional in linear spaces with applications to vector equilibria. SIAM J. Optim. 2016;28:2677-2695. Available from: https://doi.org/10.1137/16M1063575.

7. Qiu JH, He F. A general vectorial Ekeland's variational principle with a P-distance, Acta Math. Sin. (Engl. Ser.). 2013;29:16551678. Available from: https://doi.org/10.1007/s10114-0132284-z.

8. Hernández E, Rodríguez-Marín L. Nonconvex scalarization in set optimization with set-valued maps. J. Math. Anal. Appl. 2007;325:1-18. Available from: https://doi.org/10.1016/j.jmaa. 2006.01.033. 
9. Li SJ, Yang XQ, Chen GY. Nonconvex vector optimization of set-valued mappings, J. Math. Anal. Appl. 2003;283:337350. Available from: https://doi.org/10.1016/S0022-247X(02) 00410-9.

10. Geoffrion AM. Proper efficiency and the theory of vector maximization. J. Math. Anal. Appl. 1968;22:616-630. Available from: https://doi.org/10.1016/0022-247X(68)90201-1.

11. Borwein JM. Proper efficient points for maximization with respect to cones, SIAM J. Control Optim. 1977;15:57-63. Available from: https://doi.org/10.1137/0315004.

12. Benson HP. An improved definition of proper efficiency for vector maximization with respect to cones. J. Math. Anal. Appl.
1979;71:232-241. Available from: https://doi.org/10.1016/ 0022-247X(79)90226-9.

13. Anh NLH, Khanh PQ, Tung LT. Higher-order radial derivatives and optimality conditions in nonsmooth vector optimization, Nonlinear Anal.: Theory, Method and Applications. 2011;74:7365-7379. Available from: https://doi.org/10.1016/j. na.2011.07.055.

14. Anh NLH, Khanh PQ. Higher-order optimality conditions for proper efficiency in nonsmooth vector optimization using radial sets and radial derivatives. J Global Optim. 2014;58:693709. Available from: https://doi.org/10.1007/s10898-013$0077-7$. 